\title{
Information content analysis for a novel TES-based hyperspectral microwave atmospheric sounding instrument
}

Prateek Kumar Dongre, Stephan Havemann, Peter Hargrave, Angiola Orlando, Rashmikant Sudiwala, et al.

Prateek Kumar Dongre, Stephan Havemann, Peter Hargrave, Angiola Orlando, Rashmikant Sudiwala, Stafford Withington, Chris Thomas, David Goldie, "Information content analysis for a novel TES-based hyperspectral microwave atmospheric sounding instrument," Proc. SPIE 10786, Remote Sensing of Clouds and the Atmosphere XXIII, 1078608 (9 October 2018); doi: $10.1117 / 12.2500516$

SPIE. Event: SPIE Remote Sensing, 2018, Berlin, Germany 


\title{
Information content analysis for a novel TES-based Hyperspectral Microwave Atmospheric Sounding Instrument
}

\author{
Prateek Kumar Dongre*a, Stephan Havemann ${ }^{\mathrm{b}}$, Peter Hargrave ${ }^{\mathrm{a}}$, Angiola Orlando ${ }^{\mathrm{a}}$, Rashmikant \\ Sudiwala ${ }^{\mathrm{a}}$, Stafford Withington ${ }^{\mathrm{c}}$, Chris Thomas $^{\mathrm{c}}$, David Goldie ${ }^{\mathrm{c}}$ \\ ${ }^{a}$ Cardiff University, School of Physics and Astronomy \\ Queens Buildings, The Parade, Cardiff, CF24 3AA \\ ${ }^{\mathrm{b}} \mathrm{UK}$ Met Office, Satellite Applications, \\ Fitzroy Road, Exeter, EX1 3PB, UK \\ ${ }^{c}$ Quantum Sensors Group, Cavendish Laboratory, University of Cambridge, \\ JJ Thomson Ave, Cambridge CB3 0HE
}

\begin{abstract}
In context of numerical weather prediction (NWP), increased usage of satellites radiance observations from passive microwave sensors have brought significant improvements in the forecast skills. In the infrared spectral region, hyperspectral sounder instruments such as IASI have already benefitted the NWP assimilation systems, but they are useful only under clear sky conditions. Currently, microwave instruments are providing wealth of information on clouds, precipitation and surface etc., but only with limited number of channels. Furthermore, due to limited number of channels and with poor signal-to-noise ratio, existing passive microwave sensors have very poor resolution and accuracy.

We are currently developing a new microwave instrument concept, based on superconducting filterbank spectrometers, which will enable high spectral resolution observations of atmospheric temperature and humidity profiles across the microwave/sub-millimeter wavelength region with photon-noise-limited sensitivity. This study aims at investigating the information content on temperature and water-vapour that could be provided by such a hyperspectral microwave instrument under clear sky-conditions. Here, we present a new concept of Transition Edge Sensors (TESs)-based hyperspectral microwave instrument for atmospheric sounding applications. In this study, for assessing the impact of hyperspectral sampling in microwave spectral region in clear sky-conditions, we have estimated the information content as standard figure of merit called as degrees of freedom for signal (DFS). The DFS for a set of temperature and humidity sounding channels $(50-60 \mathrm{GHz}, 118 \mathrm{GHz}$ and $183 \mathrm{GHz})$ have been analyzed under the linear optimal estimation theory framework.
\end{abstract}

Keywords: Hyper-spectral Microwave, Atmospheric sounders, Information content, Temperature, Watervapour, Transition-edge Sensors

*Email: dongrep@cardiff.ac.uk; Astronomy Instrumentation Group, School of Physics and Astronomy, Cardiff University, Cardiff-CF24 3AA, Wales, United Kingdom

Remote Sensing of Clouds and the Atmosphere XXIII, edited by Adolfo Comerón, Evgueni I. Kassianov,

Klaus Schäfer, Richard H. Picard, Konradin Weber, Proc. of SPIE Vol. 10786, 1078608

(c) 2018 SPIE CCC code: $0277-786 \mathrm{X} / 18 / \$ 18 \cdot$ doi: $10.1117 / 12.2500516$ 


\section{INTRODUCTION}

Satellite observations are one of the most important sources of atmospheric information to numerical weather prediction (NWP) systems. In the past decades, there has been a significant increase in assimilation of satellite data, both from infrared and microwave sensors, to NWP models, which has brought noticeable improvements in weather forecast skills. Many Atmospheric Observing System Simulation Experiments (OSSEs) and Forecast Sensitivity of Observation (FSO) experiments have shown the positive impact of satellite data on the NWP assimilation systems [1-3]. These sensitivity experiments have also revealed that instruments such as the Advanced Microwave Sounding Unit-A (AMSU-A) and the Infrared Atmospheric Sounding Interferometer (IASI) are the major instruments, which have significantly contributed in the reduction of forecast errors for the temperature soundings [1], [4-7]. In the Infrared (IR) spectral region, hyperspectral sounders such as the Atmospheric Infrared Sounder (AIRS) (2378 channels), Cross-Track Infrared Souder (CrIS) (1305 channels) and the Infrared Atmospheric Sounding Interferometer (IASI) (8461 channels) provides high-quality and high-resolution data on temperature, humidity and other trace gases. The data from these instruments are already currently benefiting the NWP assimilation systems. But these sensors can provide useful data only under clear-sky conditions as infrared data is greatly affected by the presence of clouds. Microwave sensors have the advantage of sensing within and penetrating the clouds and therefore they can provide useful information even in the presence of clouds. Currently, existing passive microwave sensors such as Advanced Microwave Sounding Unit-A (AMSU-A), Advanced Microwave Sounding Unit-B (AMSU-B), Advanced Technology Microwave Sounder (ATMS), Microwave Humidity Sounder (MHS) and Special Sensor Microwave Imager/Sounder (SSMI/S) are providing valuable information on atmosphere, surface, the hydrometeors (rain, ice, etc.). But, vertical resolution is very poor, as all of these microwave instruments provide observations with very limited number of sounding channels (ranges from 5 to 30 channels) [8]. The radiometric noise of these microwave instruments is also a limitation on their sounding performance.

With recent technological advancements, we are now investigating a microwave radiometer with a significantly increased number of very low noise channels. The instrument concept is a new hyperspectral microwave instrument, enabled by TES-based on-chip superconducting filterbank spectrometers [9]. This technology should allow the incoming signal to be split into up to 1,000 narrow-band spectral channels. This new solution will allow full access to the 50-60 GHz atmospheric oxygen lines, which are critical for high-quality atmospheric temperature retrievals, with the potential for continuous spectral coverage up to $\sim 1 \mathrm{THz}$. This will enable a new generation of high-accuracy and high-resolution temperature and humidity sounding instruments, to improve the NWP weather forecasting skill.

Optimal design of such an instrument includes consideration of various factors such as: instrument noise, channel optimization (channel frequencies, bandwidth and spectral resolution), scanning geometrical parameters (coverage, integration time) and radiometer receiver design etc. In order to perform such optimization studies, we require an evaluation of the impact of instrument parameters on the retrieval capability. The work presented here aims at analyzing the information content (of temperature and water vapour) that could be provided by this new-advanced TES-based hyperspectral microwave instrument. We present some of our initial results of information content analysis for such an instrument. 


\section{TECHNOLOGY DEVELOPMENTS FOR ENABLING HYPERSPECTRAL MICROWAVE ATMSOPHERIC SOUNDING INSTRUMENT}

\subsection{Satellite Atmospheric Microwave Sounding Instruments}

Currently, microwave sounders are providing large amount of useful information on temperature, humidity, clouds, surface and hydrometeors (rain, ice etc.). Some of the important current microwave instruments are: AMSU-A (AMSU-B), ATMS and SSMI/S. All these instruments have relatively large radiometric noise and poor vertical resolution (because of limited number of channels ranges from 5 to 30 ) [8]. Even some of the future planned MetOp-SG microwave instruments such as: Microwave Sounder (MWS), Microwave Imager (MWI) [10] and Ice Cloud Imager (ICI) [11] will contain 10 to 25 channels. A very high-spectral resolution instrument with low noise channels in the microwave spectral region is technologically challenging. But, due to recent technological advancements, it is now feasible to design and build such an instrument. In the next section, some of the recent advancements have been discussed.

\subsection{TES-based Superconducting Filterbank Spectrometer Technology for Hyperspectral Microwave Instrument}

We are currently developing a new chip filterbank spectrometer technology based on transition edge sensors (TESs) [12-15]. Such a filter bank spectrometer has been proposed as a core enabling technology for a future hyperspectral microwave instrument for atmospheric remote sensing applications. No other group has adopted this approach. The TES's are well proven technology in many areas of observational astrophysics due to their reliability, low-noise and high-dynamic range.

Filterbank spectrometer based on Kinetic Inductance Detectors (KIDs) [16-18] are being developed in the DESHIMA [19] and SuperSpec [20] projects. It may be possible to adapt KID instruments to make atmospheric spectrometers, but they have certain shortcomings and difficulties. The TES-based technology has several advantages over KIDs such as: it's ability to work at longer wavelengths, high saturation power and ease of matching the transmission lines to the detectors.

Our filterbank spectrometer will employ an array of TES superconducting detectors to sense the power in each spectral bin. This technology will enable the operation in the very important $50-60 \mathrm{GHz} \mathrm{O}$ temperature sounding band, which is of critical importance to NWP. We aim to demonstrate, in the very near future, devices that can employ a large number of spectral channels, with spectral resolutions $(v / \Delta v)$ up to $\sim 1,000$, with photon-noise-limited sensitivity in each channel. 


\section{METHODOLOGY}

The methodology adopted for assessing the information content of atmospheric variables (temperature and humidity) from satellite microwave observations is mainly based on Optimal estimation theory (OE) [21]. Much of the theoretical background of the Optimal estimation method (OE) can be found in detail in Rodgers (2000) [21]. This theory has been used in many other information content studies [22-24]. In this section, the Bayesian framework of atmospheric inverse problems and the formulation used for the information content analysis will be described. We have used the standard NWP data assimilation notations [25] to describe the mathematical formulations of inverse problem and information content analysis.

\subsection{Linear Optimal Estimation Theory and Bayes Theorem}

Estimation of state of the atmosphere from indirect satellite measurements is an ill-posed inverse problem. For setting up this inverse problem mathematically, we have denoted the unknown state of the atmosphere (here atmospheric temperature and humidity profiles to be retrieved) as vector $\boldsymbol{x}$ and the hyperspectral satellite observations from a microwave radiometer as vector $\boldsymbol{y}$. The physics of the satellite measurement process of atmospheric state parameters (temperature and humidity) links the state vector $\boldsymbol{x}$ and the measurement vector $\boldsymbol{y}$, and this process will be described by a forward model. Mathematically, the forward model with some error in the measurement process is given by the equation (1) as:

$$
\boldsymbol{y}=H(\boldsymbol{x})+\boldsymbol{\epsilon}
$$

Where, $H$ is the observation operator or forward model operator which can be linear or non-linear. The observation operator is basically our understanding of physics involved in the satellite measurement process (in this case, it will comprise of our knowledge of atmospheric radiative transfer, and molecular spectroscopic parameters etc.), and $\epsilon$ is the measurement error (for e.g. errors in the radiative transfer, calibration errors, instrument noise and errors in the molecular spectroscopic parameters measurements etc.). In order to get the atmospheric parameters of interests, a reverse operation on the forward model equation (1) has to be performed, and this task is called as the Inverse problem. A very useful approach to this kind of atmospheric inverse problems is Bayesian approach. In this approach, the process of satellite measurement of atmospheric state vectors will be described by the probability distribution functions (PDFs) in the framework of Bayes' theorem as:

$$
p(\boldsymbol{x} \mid \boldsymbol{y})=\frac{p(\boldsymbol{y} \mid \boldsymbol{x}) p(\boldsymbol{x})}{p(\boldsymbol{y})}
$$

Where, $p(\boldsymbol{x} \mid \boldsymbol{y})$ is the posterior distribution of the state of atmosphere $\boldsymbol{x}$, given the observation $\boldsymbol{y}, p(\boldsymbol{y} \mid \boldsymbol{x})$ is the probability of the observations $\boldsymbol{y}$ given $\boldsymbol{x}, p(\boldsymbol{x})$ and $p(\boldsymbol{y})$ are the a-priori probabilities of $\boldsymbol{x}$ and $\boldsymbol{y}$ respectively, while $p(\boldsymbol{y})$ is often treated as normalization factor and it is independent of $\boldsymbol{x}$. Assuming linear observation operator $H$ and Guassianity of the probability distribution functions (PDFs), the posterior PDF $p(\boldsymbol{x} \mid \boldsymbol{y})$ is determined by the multiplication of likelihood PDF of observations $p(\boldsymbol{y} \mid \boldsymbol{x})$ and an a priori PDF $p(\boldsymbol{x})$ of atmospheric state estimate $\boldsymbol{x}_{b}$. In multi-variate case, the formulation of posterior PDF $p(\boldsymbol{x} \mid \boldsymbol{y})$ will also involves the error covariance matrices of the background state, $\boldsymbol{B}$, and the measurement errors (that 
can originate from observations and modelling), which is equal to $\boldsymbol{R}=\boldsymbol{E}+\boldsymbol{F}$, where $\boldsymbol{R}$ is the observationerror covariance matrix, $\boldsymbol{E}$ is the Instrument noise and $\boldsymbol{F}$ is the representativity errors. The posterior PDF $p(\boldsymbol{x} \mid \boldsymbol{y})$ is given by the equation (3) as:

$$
p(\boldsymbol{x} \mid \boldsymbol{y})=\exp \left[-\frac{1}{2}\{\boldsymbol{y}-H(\boldsymbol{x})\}^{T} \boldsymbol{R}^{-1}\{\boldsymbol{y}-H(\boldsymbol{x})\}-\frac{1}{2}\left\{\boldsymbol{x}-\boldsymbol{x}_{\boldsymbol{b}}\right\}^{T} \boldsymbol{B}^{-1}\left\{\boldsymbol{x}-\boldsymbol{x}_{\boldsymbol{b}}\right\}\right]
$$

In the linear case, the optimal analysis state $\boldsymbol{x}_{a}$ can be derived as the mathematical expression given in the equation (4):

$$
\boldsymbol{x}_{a}=\boldsymbol{x}_{b}+\boldsymbol{A} \boldsymbol{H}^{\boldsymbol{T}} \boldsymbol{R}^{-1}\left\{\boldsymbol{y}-H\left(\boldsymbol{x}_{b}\right)\right\}
$$

and the posterior error covariance matrix $\boldsymbol{A}$ (which is often termed as analysis-error covariance matrix) is given by the following equation (5):

$$
\boldsymbol{A}=\left(\boldsymbol{B}^{-1}+\boldsymbol{H}^{T} \boldsymbol{R}^{-1} \boldsymbol{H}\right)^{-1}
$$

Where $\boldsymbol{H}$ is the Jacobian matrix, which contains the first-order derivatives of each measurement with respect to each element of the state vector $\boldsymbol{x}, \boldsymbol{R}$, is the observation error-covariance matrix which comprises of different types of errors such as: radiative transfer errors, instrument noise, representativity errors etc. But in this study, only instrument noise has been taken into account as a description of $\boldsymbol{R}$ matrix. The OE approach combines the background information (represented by the background error-covariance matrix $\boldsymbol{B})$ with the satellite radiance measurements error information (represented by the observation errorcovariance matrix $\boldsymbol{R}$ ) to estimate the analysis $\boldsymbol{A}$. These three important components of information content analysis: background error-covariance matrix $\boldsymbol{B}$, Observation error-covariance matrix $\boldsymbol{R}$ and Jacobian matrix $\boldsymbol{H}$ has been described in detail in the section 5 .

\subsection{Information Content}

The information content of satellite measurements can be estimated by comparing background (a-priori errors) with the analysis errors. The comparison between background errors and analysis errors provides an understanding of extent of information that can be induced by the satellite measurements as compared to the background information. Quantitatively, the information content can be provided by the metric called as the Degrees of Freedom for Signal (DFS) [21].The DFS is a standard scalar metric widely used in the NWP community as a measure of information content of satellite radiances [26-29]. It has also been used in many other previous information content and channel selection studies [22], [23], [26], [27], [30]. It can be mathematically expressed as given in the equation (6):

$$
D F S=d_{s}=\operatorname{tr}\left(\boldsymbol{I}-\boldsymbol{A} \boldsymbol{B}^{-1}\right)
$$

Where, $d_{s}$ is the degrees of freedom for signal and $t r$ stands for the Trace of the matrix, which is equivalent to the sum of the elements on the main diagonal of the matrix. 


\section{MODELS AND DATA}

\subsection{Atmospheric Profiles}

A set of eight atmospheric profiles have been used in this study, which represents the state of the atmosphere in terms of profiles of temperature, water vapour and trace gases etc. Seven of these profiles (Profile 2 to 8) have been extracted directly from the Met Office Unified Model (UM) in March 2012 and Profile 1 is the US standard atmosphere. The profiles are on 70 model levels from a range of different longitudes and latitudes.

\subsection{Radiative Transfer Model}

For calculating the sensitivity of frequencies towards atmospheric variables of temperature and water vapour (which are also called as the Jacobians), a radiative transfer model is required. For each atmospheric profile, these Jacobians have been calculated using a model called as the Havemann-Taylor Fast Radiative Transfer Code (HT-FRTC) [31]. This radiative transfer model is designed to simulate the radiation from microwave to ultraviolet spectral range. It is a principal component (PC) based model and it can cover the whole electromagnetic spectrum represented by their principal components. Various functionalities of this model have been fully described in [31]. The model can be used in either in fast or in line-by-line (LBL) mode. But, for this study, line-by-line mode of the HT-FRTC has been used and it has been denoted as line-HT-FRTC. The line-HT-FRTC model takes account of satellite instrument parameters (such as: channels frequencies, bandwidth and spectral resolution) by the use of a sensor-specific file, which makes it highly flexible and appropriate for investigation of future planned meteorological satellite instruments or newly proposed mission concepts. The line-HT-FRTC has been used to calculate the analytic Jacobians for temperature and humidity. The HT-FRTC model has been used in several other airborne and spaceborne remote sensing studies (recently, it has been used for investigating a new high-resolution infrared instrument, Laser Heterodyne Radiometer (LHR) for numerical weather prediction application applications [24]. The line-HT-FRTC model has also been compared and validated with other radiative transfer models [32].

\section{COMPONENTS OF INFORMATION CONTENT ANALYSIS}

In this section, we have described the important components which is required to perform the information content analysis. As mentioned earlier in the section, we have used the standard NWP notation [25] to describe the components of information analysis in matrix notations. This will include the description of background error-covariance matrix $\boldsymbol{B}$, observation error-covariance matrix $\boldsymbol{R}$, Jacobian matrix $\boldsymbol{H}$ and analysis error-covariance matrix $\boldsymbol{A}$. The mathematical relationship between these three components has been described in the equation (5).

\subsection{Background error-covariance matrices $B$}

Bayes' theorem based OE method requires the knowledge of the a priori information of the atmosphere, which is needed for the calculation of the information content (as can be seen from the equation (2) and (6)). The a priori information mainly comes from the short numerical forecast, also called as the background state $\boldsymbol{x}_{\boldsymbol{b}}$. The background state $\boldsymbol{x}_{\boldsymbol{b}}$ has to be known together with its associated errors, also called as background errors, which are represented by the background error-covariance matrices $\boldsymbol{B}$. The $\boldsymbol{B}$ matrix is complex in structure and it is the combination of background error standard deviation and 
vertical error correlations. It is an important component of the analysis as the information content will be calculated with respect to the background information [23-33]. As this study is mainly focused on temperature and water vapour measurements, the $\boldsymbol{B}$ matrix used here only comprises of temperature $(T)$ and humidity $(q)$ errors. The structure of background error covariance matrix of temperature and humidity are shown in Figure 1.
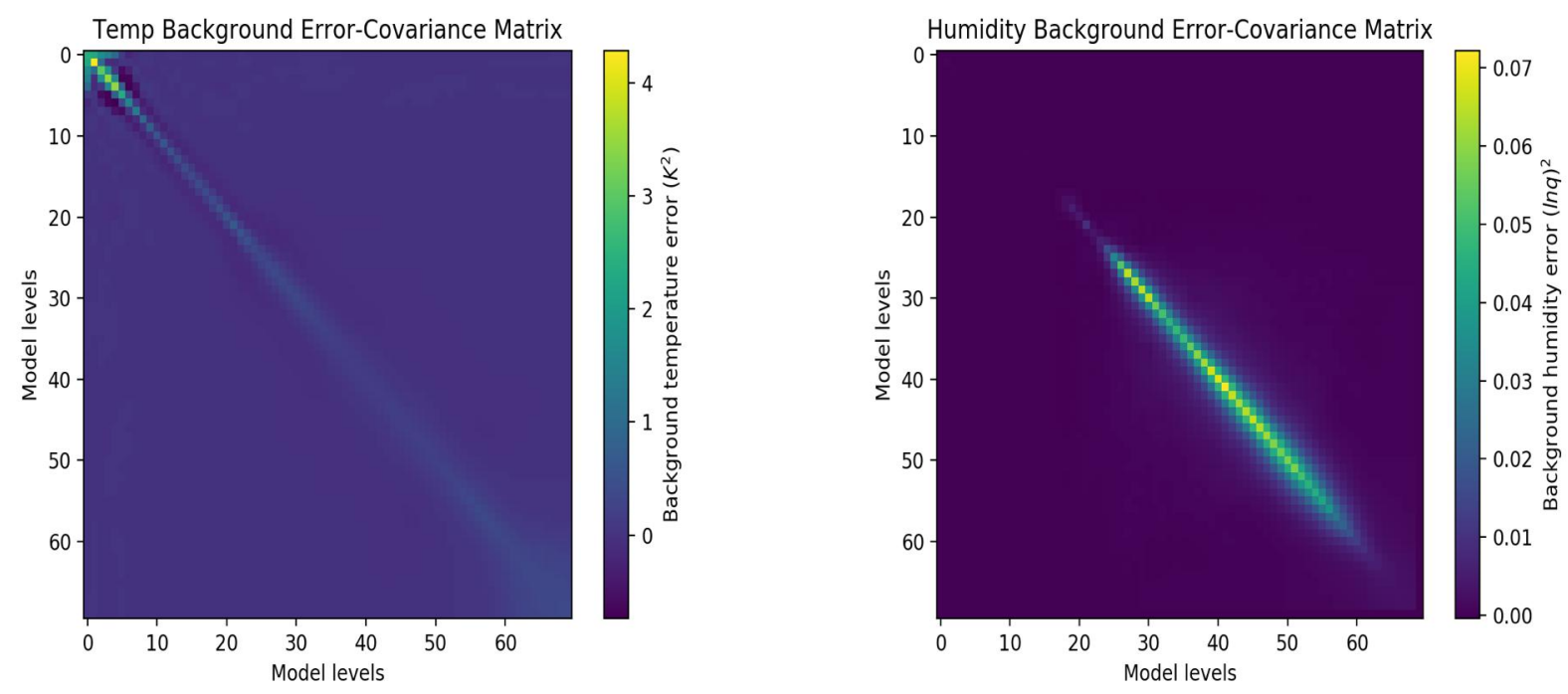

Figure 1: Background error-covariance matrices (in left, for temperature (T) and in right, for humidity (q)), which is used in this information content study

The $\boldsymbol{B}$ matrix used in this study is a 1D-representation of the 4D-Var error covariance matrix in operational use at the Met Office in March 2012. These 1D Var matrices were derived from the operational Met Office 4D-Var error covariance model. The same $\boldsymbol{B}$ matrix has been used in other information content and sensitivity studies for e.g. in Smith (2015) [34] and in Eyre and Hilton (2013) [35]. More mathematical details about this $\boldsymbol{B}$ matrix can be found in Eyre and Hilton (2013) [35].

\subsection{Jacobian Matrix $H$}

Another important component of an information content analysis is the Jacobian matrix $\boldsymbol{H}$. The Jacobians are the sensitivity of the brightness temperature with respect to the change in the quantity of the atmospheric variables of interest. The Jacobians for temperature and water vapour has been calculated analytically by using line-HT-FRTC model. These temperature and humidity Jacobians forms the elements of the $\boldsymbol{H}$ matrix, which can be mathematically represented as $\partial T_{b} / \partial T_{i}$ (where $T_{b}$ is the brightness temperature and $T_{i}$ is the temperature in $K$ at level $i$ ) and as $\partial T_{b} / \partial q_{i}$ (where $q_{i}$ is the humidity at level $i$ ) respectively. 


\section{EXPERIMENTAL FRAMEWORK: ANALYSIS OF SENSITIVITY OF INFORMATION CONTENT}

In this section, a framework is designed to perform the experiments to test the sensitivity of information content towards various factors. The components, background error covariance matrix $\boldsymbol{B}$, Observation error covariance matrix $\boldsymbol{R}$ and Jacobian matrix $\boldsymbol{H}$ have been systematically combined to analyze the information content on temperature and water vapour. Firstly, a set of candidate channels (and frequency range) have been selected initially for performing the sensitivity experiments (the selection of channels and sensitivity experiments has been discussed in detail in the following sections 6.1, 6.2 and 6.3). Some of our initial results of the information content analysis (with focus on two important factors of spectral resolution and instrument noise) have been discussed and presented here in the following subsequent sections.

\subsection{Absorption bands and Candidate channel frequencies}

The microwave absorption spectrum is relatively less complex as compared to the IR spectrum, as there are only few spectral features that absorbs in microwave spectral region. Atmospheric gases such as, oxygen $\left(\mathrm{O}_{2}\right)$ and water vapour $\left(\mathrm{H}_{2} \mathrm{O}\right)$ strongly absorbs in the microwave spectrum. Most of these $\mathrm{O}_{2}$ and $\mathrm{H}_{2} \mathrm{O}$ absorption lines in this region are isolated. In NWP context, the $\mathrm{O}_{2}$ and $\mathrm{H}_{2} \mathrm{O}$ absorption bands are important for their application in the retrievals of temperature and humidity. Hyper-spectral sampling in IR (as in IASI instrument) strongly bring improvements in the retrievals. On the similar line, to analyze the potential performance of hyperspectral satellite microwave measurements in the $50-200 \mathrm{GHz}$ frequency range, we have selected a set of candidate frequency bands in this range (indicated in Table 1). The frequency bands have been selected based on the application of the $\mathrm{O}_{2}$ (for temperature sounding) and $\mathrm{H}_{2} \mathrm{O}$ (for humidity sounding) absorption bands.

$\mathrm{O}_{2}$ bands in $50-60 \mathrm{GHz}$ and in $118 \mathrm{GHz}$ have been extensively used by many current passive microwave sensors for temperature information (such as AMSU-A, ATMS and SSMI/S)) and $\mathrm{H}_{2} \mathrm{O} 183 \mathrm{GHz}$ absorption band for humidity information (such as AMSU-B, Microwave Humidity Sounder (MHS)). For this study, we have mainly focused our analysis initially around 50-60, $118 \mathrm{GHz} \mathrm{O}_{2}$ band (for T-sounding) and around $183 \mathrm{GHz}$ band (for q-sounding). An overview of three frequency bands considered in this study are represented in the Table 1 . The channels in each spectral range has been distributed according to their uniform bandwidths $(\mathrm{BW})$. The distribution of spectral channels follows the equation: $f_{i}=f_{\min }+$ $(i-1) d$, Where, $f_{\text {min }}=$ Starting minimum frequency (in each frequency range), $d=$ channel spacing, and is given by, $d=\left(f_{\max }-f_{\min }\right) /(n-1), n=$ number of channels.

Different instrument configurations have been presented in the Table 1 for conducting the information content sensitivity experiments. A spectral resolution (with $\mathrm{N}_{1}$ number of channels) has been assumed initially as "low spectral resolution". A multiplication factor of 2 is applied to increase the spectral resolution as "high-spectral resolution" (with $\mathrm{N}_{2}$ number of channels). Specifically, for investigating the impact of instrument noise, we have designed two instrument configurations based on the types of radiometer technologies. Two different types of technologies are considered - Heterodyne radiometer receiver based system and superconducting transition edge sensor based radiometer receiver system. The instrument noise figures for these two different types of microwave radiometer technology has been

calculated and represented in the Table 1 as "IN TES", for superconducting TES-based radiometer receiver system (low instrument noise system) and as "IN $N_{H E T}$ " for the heterodyne radiometer receiver (relatively high instrument noise systems). 
Table 1: Frequency bands selected for analyzing the performance of hyperspectral measurements of temperature ( $\mathrm{T})$ and humidity (q). " $R_{1}$ " indicates the low spectral resolution and " $R_{2}$ ", indicates high-spectral resolution. Channel configurations with two noise characteristics for investigating the effect of instrument noise on information content of temperature and water vapour. The range of instrument noise (estimated for TES-based radiometer receivers) is given for all the channels present in that frequency band.

\begin{tabular}{|c|c|c|c|c|c|c|c|}
\hline \multirow[t]{2}{*}{$\begin{array}{c}\text { Frequency } \\
\text { Band } \\
\text { Name }\end{array}$} & \multicolumn{2}{|c|}{$\begin{array}{l}\text { Frequency } \\
\text { Range (in } \\
\text { GHz) }\end{array}$} & \multirow[t]{2}{*}{$\begin{array}{c}\text { Bandwidth } \\
\text { (BW) } \\
\text { (MHz) }\end{array}$} & \multicolumn{2}{|c|}{$\begin{array}{l}\text { Number of sounding } \\
\text { channels }\end{array}$} & \multicolumn{2}{|c|}{$\begin{array}{c}\text { Estimated Instrument } \\
\text { Noise (mK)- based on } \\
\text { technology }\end{array}$} \\
\hline & $f_{\min }$ & $f_{\max }$ & & $\begin{array}{c}\text { Resolution } \\
\left(\mathbf{R}_{1}\right) \\
\mathbf{N}_{1}\end{array}$ & $\begin{array}{c}\text { Resolution } \\
\left(\mathbf{R}_{2}\right) \\
\mathbf{N}_{2}\end{array}$ & $\begin{array}{l}(\text { IN } \\
\text { HET }) \\
\text { Heterodyne } \\
\text { receiver- } \\
\text { based } \\
\text { systems } \\
(\mathrm{mK}) \text { - }\end{array}$ & $\begin{array}{c}\left(\mathrm{IN}_{\mathrm{TES}}\right) \\
\text { TES-based } \\
\text { radiometer } \\
\text { receiver } \\
\text { system } \\
\text { noise range } \\
(\mathrm{mK}) \\
\end{array}$ \\
\hline \multirow{2}{*}{$\begin{array}{c}\mathrm{O}_{2} 50-60 \\
\text { GHz Band }\end{array}$} & 50.3 & 57.8 & \multirow{2}{*}{100} & 50 & 100 & 400 & $2.01-2.21$ \\
\hline & 60.7 & 67.9 & & 50 & 100 & 400 & $2.21-2.34$ \\
\hline $\begin{array}{c}\mathrm{O}_{2} 118 \\
\text { band } \mathrm{GHz}\end{array}$ & 113.7 & 123.7 & 200 & 51 & 102 & 420 & $2.14-2.23$ \\
\hline \multirow{2}{*}{$\begin{array}{c}\mathrm{H}_{2} \mathrm{O} 183 \\
\mathrm{GHz}\end{array}$} & \multirow[t]{2}{*}{173.3} & \multirow[t]{2}{*}{193.3} & \multirow[t]{2}{*}{400} & 51 & 102 & 400 & $1.81-1.96$ \\
\hline & & & & $\mathrm{N}_{1 \mathrm{t}}=202$ & $\mathrm{~N}_{2 \mathrm{t}}=404$ & & \\
\hline
\end{tabular}

\subsection{Sensitivity Study 1: Effect of high-spectral resolution on the Jacobians}

In this section, we have conducted a sensitivity study to examine the effect of the high-spectral resolution on the Jacobians. We aim here to analyze the density and spread of the Jacobians in the levels of the atmosphere (which can give us an idea about the distribution of the information content). We have assumed two spectral resolutions, one with lower number of channels (low-spectral resolution), $\mathrm{N}_{1}$ and the other with higher number of channels $\mathrm{N}_{2}$ (high spectral resolution), where $\mathrm{N}_{2}$ $=2 \mathrm{~N}_{1}$. The channel frequencies, their distribution and the bandwidth (BW) corresponds to $\mathrm{N}_{1}$ and $\mathrm{N}_{2}$, can be seen in the Table 1. We have calculated the Jacobians for these two spectral resolutions using line-HT-FRTC model: low spectral resolution $\mathrm{R}_{1}$ (with $\mathrm{N}_{1}=202$ channels) and for high spectral resolutions $R_{2}$ (with $\mathrm{N}_{2}=404$ channels). Temperature Jacobians for the spectral resolutions $R_{1}$ and $R_{2}$ are represented in the Figure 2. 
The effect of increase in number of channel can be seen on the density and vertical spread of Jacobians in the Figure 2. High-spectral resolution significantly increases the sampling. It can been seen from the Figure 2, that there are some high-peaking Jacobians that are sounding in the upper part of atmosphere, due to increase in number of channels. High-spectral resolution is able to capture relatively more information from the upper part of the atmosphere, which is not possible with lowspectral resolution. Figure 2, also indicates that, the density of Jacobians increases significantly in almost every level of atmosphere, and this can be attributed to the increase in number of channels.
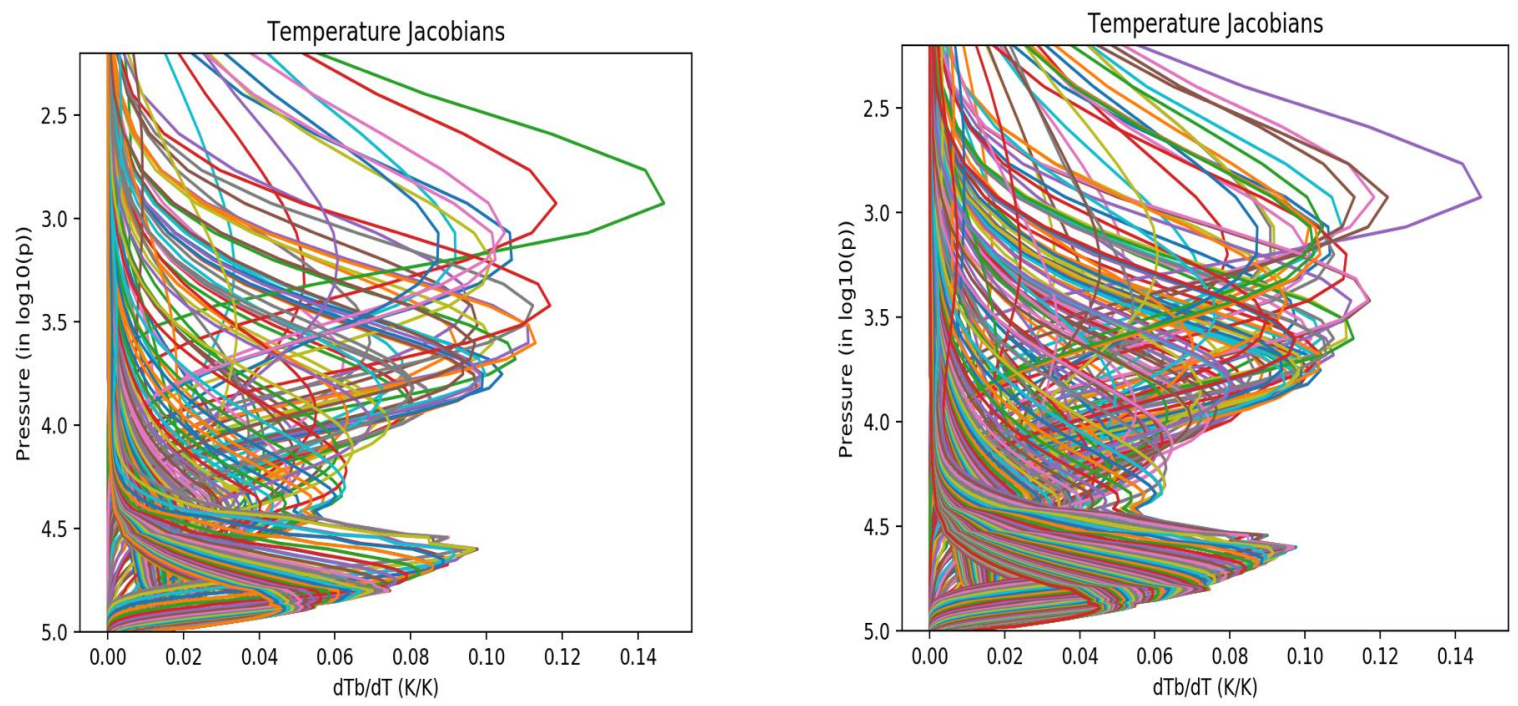

Figure 2: Temperature Jacobians for two spectral resolutions - in left, low spectral resolution (with $\mathrm{N}_{1}=202$ channels) and in right, for high-spectral resolution (with $\mathrm{N}_{2}=404$ Channels)

\subsection{Sensitivity Study 2: Effect of low instrument noise}

The instrument noise appears in the form of Instrument noise error covariance matrix $\boldsymbol{R}$ in the analysis error covariance matrix equation (5), and hence it is an important factor in the calculation of information content (as can be seen in the equation (6)). It's therefore important to test the sensitivity of the information content towards the instrument noise assumptions. In this second sensitivity experiment, we have studied the effect of instrument noise on the information content (of temperature and water vapour) and on the retrieval error reduction. Microwave instruments such as the AMSU-A, SSMI/S and ATMS, have relatively large radiometric noise across all the channels that roughly ranges from $0.2-3.0 \mathrm{~K}$. This poses a limitation on the sounding performance of these instruments. But, with, the TES-based filterbank spectrometer technology, extremely low instrument noise can be achieved for a microwave radiometer with wide spectral range and high number of channels. Two instrument configurations (having same set and $\mathrm{N}_{1}$ number of channels) but with different noise properties and values (as given in Table 1 for Heterodyne-based radiometer and TES-based radiometer) has been compared, and the results are represented in terms of retrieval error reduction (as shown in Figure 3) and information content (calculated as DFS, as shown in Figure 4). Figure 3 represents the retrieval errors (for $\mathrm{T}$ and q) estimated for the Heterodyne-based and TES-based radiometers (corresponding noise figures are given in the Table 1). It 
can be seen from the Figure 3, that the retrieval errors have been significantly reduced in case TES-based radiometer system as compared to the Heterodyne radiometer system.
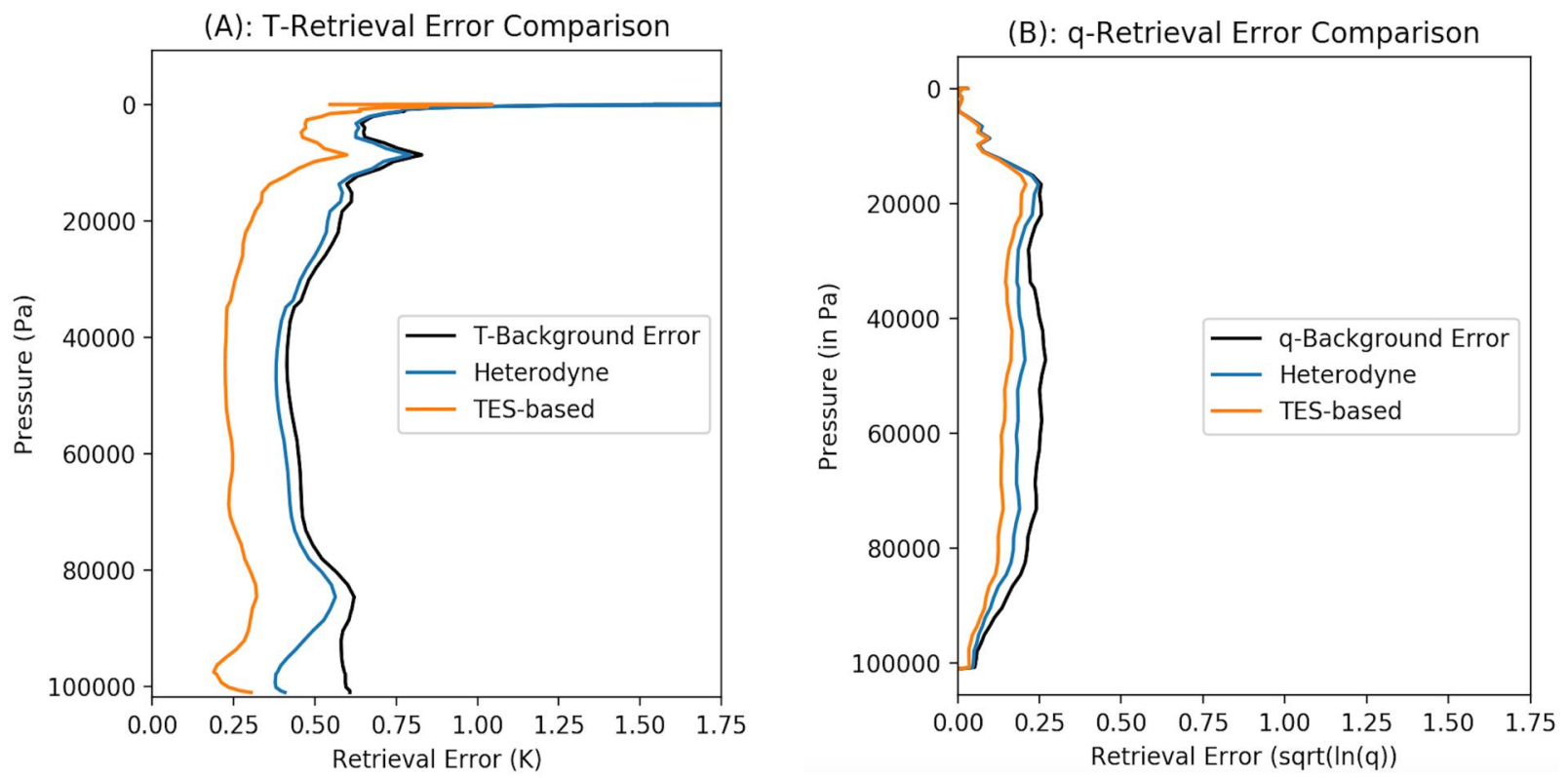

Figure 3: Effect of the instrument noise on retrieval error statistics of (A) Temperature and (B) Water vapour, as compare to the a-priori (or background information). The plots are represented for two instruments (with different noise properties as represented in the Table 1)- Heterodyne radiometer reciever (blue line) and TES-based radiometer reciever (orange line)

Similar results have been obtained in terms of information content (which is calculated as Average DFS over all 8 atmospheric profiles). Figure 4 shows the information content (as average DFS) for

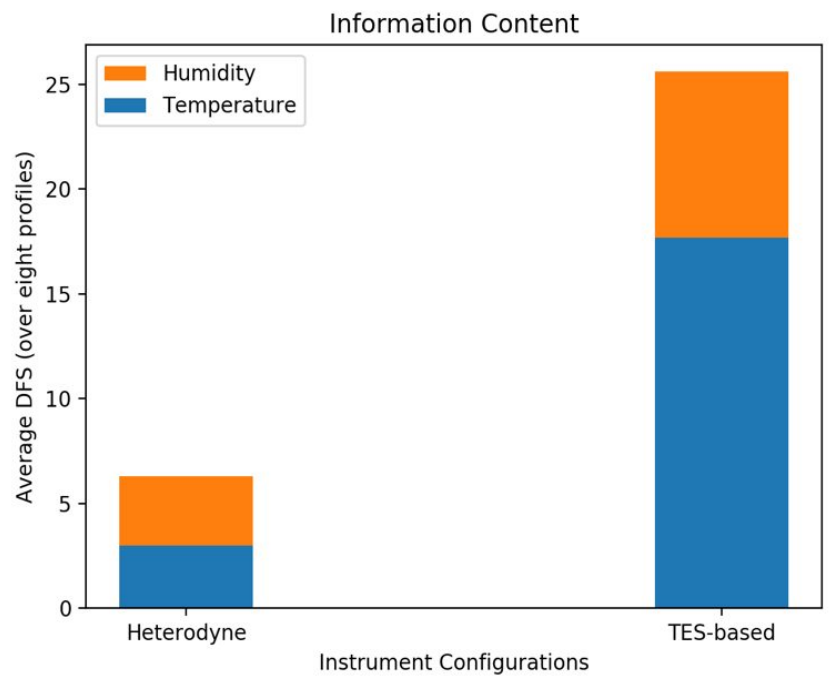

Figure 4: Estimation of information content: Comparison of the information content (calculated as DFS) on temperature and humidity provided by a Heterodyne and a TES-based radiometer receiver system for particular set of channel configuration denoted as $\mathrm{N}_{1}$ with different noise properties (as given in the Table 1). 
two hyperspectral instrument configurations (Heterodyne and TES-based instrument). Figure 4 clearly shows that the information content provided by a TES-based radiometer is significantly larger than that by a Heterodyne-based radiometer, which can be mainly attributed due to the low instrument noise (possible by TES-based filterbank spectrometer technology).

\section{DISCUSSIONS AND CONCLUSIONS}

In this article, we have presented the recent technological advancements that can realize a microwave radiometer with significantly increased number of low-noise channels. This new hyperspectral microwave instrument will be enabled by the use of transition edge sensors (TESs) based on-chip superconducting filterbank spectrometers. The advantages of this new state-of-the-art technology such as: extremely low instrument noise and flexibility in increasing in number of channels, makes it attractive and worth investigating for earth observation applications. For demonstrating the benefits of the TES-based superconducting filterbank spectrometer technology in atmospheric remote sensing applications, we have conducted an information content study. The objective of this study was to analyze the information content on temperature and water vapour and to investigate the reduction in retrieval errors that could be provided by this new TES-based hyperspectral microwave atmospheric sounding instrument. An information content analysis framework has been developed to study the effect of high-spectral resolution and instrument noise on the information content and retrieval error reduction. The framework of information content analysis involves various inputs (Jacobians (simulations performed on a set of atmospheric profiles by the use of a line-HT-FRTC model), Background information and Instrument noise).

We have specifically conducted two experiments to analyze the sensitivity of information content towards spectral resolution and instrument noise. In that context, we have presented some of our initial results of these information content sensitivity experiments. The results shows that high-spectral resolution can sample the atmosphere well and capable of extracting small detail information (which is not possible with low-spectral resolution). It also indicates that, the denser sampling along the microwave absorption lines could reduce the retrieval errors of temperature and humidity. From our second experiment, we have analyzed the effect of instrument noise by comparing two instrument configurations (Heterodyne and TESbased radiometer receiver) with same set of channels, but with different instrument noise figures. The results show us that an increase in information content is possible by achieving low instrument noise (using low noise property of a TES-based radiometer systems). Similar results have been obtained in terms of retrieval errors (of temperature and humidity) reduction.

\section{ACKNOWLEDGEMENTS}

We are sincerely thankful to The Commonwealth Scholarship Commission in the UK (CSC) for Prateek Kumar Dongre's PhD funding (CSC Reference Number: INCS-2016-214). Also, we would like to thank the UK Met Office and the European Center for Medium-Range Weather Forecasts (ECMWF, Reading) for their support and guidance. The authors are also grateful to Dr. Stuart Newman (Met Office) and Dr. William Bell (ECMWF, Reading) for their useful discussions and valuable suggestions. 


\section{REFERENCES}

[1] S. English et al., "Impact of satellite data," ECMWF Technical Memorandum, 2013.

[2] A. C. Lorenc and R. T. Marriott, "Forecast sensitivity to observations in the Met Office Global numerical weather prediction system," Q. J. R. Meteorol. Soc., vol. 140, no. 678, pp. 209-224, 2014.

[3] T. McNally, Observing system experiments to assess the impact of possible future degradation of the Global Satellite Observing Network. 2012.

[4] Y. Zhu and R. Gelaro, "Observation Sensitivity Calculations Using the Adjoint of the Gridpoint Statistical Interpolation (GSI) Analysis System,” Mon. Weather Rev., vol. 136, no. 1, pp. 335-351, Jan. 2008.

[5] C. Cardinali, "Monitoring the observation impact on the short-range forecast," Q. J. R. Meteorol. Soc., vol. 135, no. 638, pp. 239-250, Jan. 2009.

[6] A. D. Collard and A. P. McNally, "The assimilation of infrared atmospheric sounding interferometer radiances at ECMWF," Q. J. R. Meteorol. Soc., vol. 135, no. 641, pp. 1044-1058, 2009.

[7] G. Radnoti, P. Bauer, A. McNally, and C. Cardinali, "ECMWF Study on the Impact of Future Developments of the Space-based Observing System on Numerical Weather Precition," 2010.

[8] S. A. Boukabara and K. Garrett, "Benefits of a hyperspectral microwave sensor: Applications in environmental monitoring and weather forecasting," in Proceedings of IEEE Sensors, 2011, pp. 18811884.

[9] P. Hargrave, S. Withington, S. A. Buehler, L. Kluft, B. Flatman, and P. Kumar Dongre, “ THz spectroscopy of the atmosphere for climatology and meteorology applications ," 2017, vol. 10210, pp. 1021010-1021013.

[10] G. Alberti, A. Memoli, ... G. P.-A. in R., and undefined 2012, "TWO Microwave Imaging radiometers for MetOp Second Generation," ieeexplore.ieee.org.

[11] V. Kangas, S. D’Addio, U. Klein, ... M. L.-... and R. S., and undefined 2014, "Ice cloud imager instrument for MetOp Second Generation," ieeexplore.ieee.org.

[12] K. Irwin, G. H.-C. particle detection, and undefined 2005, "Transition-edge sensors," Springer.

[13] D. J. Goldie, A. V. Velichko, D. M. Glowacka, and S. Withington, "Ultra-low-noise MoCu transition edge sensors for space applications," J. Appl. Phys., vol. 109, no. 8, p. 084507, Apr. 2011.

[14] D. Osman, S. Withington, D. J. Goldie, and D. M. Glowacka, "Transition edge sensors with few-mode ballistic thermal isolation," J. Appl. Phys., vol. 116, no. 6, p. 064506, Aug. 2014.

[15] D. J. Goldie, K. Rostem, and S. Withington, "High resolution on-chip thermometry using a microstripcoupled transition edge sensor," J. Appl. Phys., vol. 108, no. 1, p. 014505, Jul. 2010.

[16] P. Day, H. LeDuc, B. Mazin, A. V.- Nature, and undefined 2003, "A broadband superconducting detector suitable for use in large arrays," nature.com.

[17] S. Doyle, P. Mauskopf, J. Naylon, A. Porch, and C. Duncombe, "Lumped Element Kinetic Inductance Detectors," J. Low Temp. Phys., vol. 151, no. 1-2, pp. 530-536, Apr. 2008.

[18] J. J. A. Baselmans et al., "A kilo-pixel imaging system for future space based far-infrared observatories using microwave kinetic inductance detectors," Astron. Astrophys., vol. 601, p. A89, May 2017.

[19] A. Endo, ... J. B.-, and Far-Infrared, and undefined 2012, "Development of DESHIMA: a redshift machine based on a superconducting on-chip filterbank," spiedigitallibrary.org.

[20] E. Shirokoff et al., "Design and Performance of SuperSpec: An On-Chip, KID-Based, mm-Wavelength Spectrometer," J. Low Temp. Phys., vol. 176, no. 5-6, pp. 657-662, Sep. 2014.

[21] C. Rodgers, Inverse methods for atmospheric sounding: theory and practice. 2000.

[22] F. Aires et al., "Microwave hyperspectral measurements for temperature and humidity atmospheric profiling from satellite: The clear-sky case," J. Geophys. Res. Atmos., vol. 120, no. 21, p. 11,334-11,351, Nov. 2015.

[23] J. Mahfouf, C. Birman, F. Aires, C. Prigent, E. Orlandi, and M. Milz, "Information content on temperature and water vapour from a hyper-spectral microwave sensor," Q. J. R. Meteorol. Soc., vol. 
141, no. 693, pp. 3268-3284, 2015.

[24] F. Smith, S. Havemann, A. Hoffmann, W. Bell, D. Weidmann, and S. Newman, "Evaluation of Laser Heterodyne Radiometry for Numerical Weather Prediction Applications," Q. J. R. Meteorol. Soc., Jun. 2018.

[25] K. Ide, P. Courtier, M. Ghil, A. L.-J. of the Meteorological, and undefined 1997, "Unified Notation for Data Assimilation: Operational, Sequential and Variational (gtSpecial IssueltData Assimilation in Meteology and Oceanography: Theory and," jstage.jst.go.jp.

[26] F. Rabier, N. Fourrie, D. Chafai, and P. Prunet, "Channel selection methods for Infrared Atmospheric Sounding Interferometer radiances," Q. J. R. Meteorol. Soc., vol. 128, no. 581, pp. 1011-1027, 2002.

[27] A. E. Lipton, "Satellite sounding channel optimization in the microwave spectrum," IEEE Trans. Geosci. Remote Sens., vol. 41, no. 4, pp. 761-781, Apr. 2003.

[28] N. Fourrié and J.-N. Thépaut, "Evaluation of the AIRS near-real-time channel selection for application to numerical weather prediction," Q. J. R. Meteorol. Soc., vol. 129, no. 592, pp. 2425-2439, Jul. 2003.

[29] A. D. Collard, "Selection of IASI channels for use in numerical weather prediction," Q. J. R. Meteorol. Soc., vol. 133, no. 629, pp. 1977-1991, Oct. 2007.

[30] C. Birman, J.-F. Mahfouf, M. Milz, J. Mendrok, S. A. Buehler, and M. Brath, "Information content on hydrometeors from millimeter and sub-millimeter wavelengths," Tellus A Dyn. Meteorol. Oceanogr., vol. 69 , no. 1, p. 1271562, Jan. 2017.

[31] S. Havemann, "The development of a fast radiative transfer model based on an empirical orthogonal functions (EOF) technique," 2006, vol. 6405, p. 64050M.

[32] H. H. Aumann et al., "Evaluation of Radiative Transfer Models With Clouds," J. Geophys. Res. Atmos., vol. 123, no. 11, pp. 6142-6157, Jun. 2018.

[33] R. Bannister, "A review of forecast error covariance statistics in atmospheric variational data assimilation. II: Modelling the forecast error covariance statistics," Q. J. R. Meteorol., 2008.

[34] F. Smith, "Improving the information content of IASI assimilation for numerical weather prediction," 2015.

[35] J. R. Eyre and F. I. Hilton, "Sensitivity of analysis error covariance to the mis-specification of background error covariance," Q. J. R. Meteorol. Soc., vol. 139, no. 671, pp. 524-533, Jan. 2013. 